\title{
Enhancement of chemotherapy by manipulation of tumour pH
}

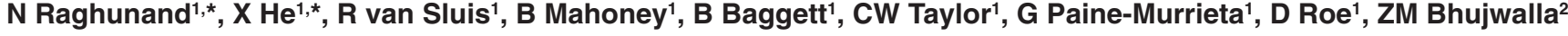 \\ and RJ Gillies ${ }^{1}$
}

${ }^{1}$ Arizona Cancer Center, 1501 N Campbell Avenue, Tucson, AZ 85724-5024, USA; '2Department of Radiology, Johns Hopkins School of Medicine, Baltimore, MD, USA

\begin{abstract}
Summary The extracellular (interstitial) $\mathrm{pH}(\mathrm{pHe}$ ) of solid tumours is significantly more acidic compared to normal tissues. In-vitro, low pH reduces the uptake of weakly basic chemotherapeutic drugs and, hence, reduces their cytotoxicity. This phenomenon has been postulated to contribute to a 'physiological' resistance to weakly basic drugs in vivo. Doxorubicin is a weak base chemotherapeutic agent that is commonly used in combination chemotherapy to clinically treat breast cancers. This report demonstrates that MCF-7 human breast cancer cells in vitro are more susceptible to doxorubicin toxicity at $\mathrm{pH} \mathrm{7.4,} \mathrm{compared} \mathrm{to} \mathrm{pH}$ 6.8. Furthermore ${ }^{31} \mathrm{P}$-magnetic resonance spectroscopy (MRS) has shown that the pHe of MCF-7 human breast cancer xenografts can be effectively and significantly raised with sodium bicarbonate in drinking water. The bicarbonate-induced extracellular alkalinization leads to significant improvements in the therapeutic effectiveness of doxorubicin against MCF-7 xenografts in vivo. Although physiological resistance to weakly basic chemotherapeutics is well-documented in vitro and in theory, these data represent the first in vivo demonstration of this important phenomenon.
\end{abstract}

Keywords: doxorubicin; acid-base balance; chemotherapy; MRS; bicarbonate

In the early part of this century, Otto Warburg postulated that tumours were acidic because the rate at which they produced lactic acid was 'remarkable' (Warburg, 1956). Subsequent measurement of $\mathrm{pH}$ in a variety of solid tumours with microelectrodes apparently confirmed this hypothesis (Wike-Hooley et al, 1984). It is assumed that microelectrode measurements primarily interrogate extracellular (interstitial) volumes. However, in-vivo ${ }^{31} \mathrm{P}$ magnetic resonance spectroscopy (MRS) of endogenous intracellular inorganic phosphate $(\mathrm{Pi})$ measures an intracellular $\mathrm{pH}(\mathrm{pHi})$ which is neutral-to-alkaline in the same or similar tumours (Griffiths, 1991; Negendank, 1992). Thus, the apparent acidity of the extracellular (interstitial) $\mathrm{pH}(\mathrm{pHe})$ does not translate to an acidic $\mathrm{pHi}$. Recently, ${ }^{31} \mathrm{P}$ MRS methods have been developed to simultaneously measure the $\mathrm{pHi}$ and $\mathrm{pHe}$ of tumours using endogenous $\mathrm{Pi}$ and exogenous 3-aminopropylphosphonate (3-APP), respectively (Gillies et al, 1994). This method has clearly shown, in a number of solid tumour xenografts, that the pHi of tumour cells is neutral-alkaline, while the pHe of the same tumours is acidic (McCoy et al, 1995; Raghunand et al, 1999a). Thus, solid tumours generally have substantial acid-outside $\mathrm{pH}$ gradients.

Acid-outside $\mathrm{pH}$ gradients have a number of important sequellae that are germane to cancer, including a theoretically reduced partitioning of weakly basic chemotherapeutic drugs into the relatively alkaline cells. This phenomenon has its basis in the 'ion-trapping' hypothesis wherein weak bases partition into, and are sequestered by, acidic compartments, such as the extracellular fluid (Roos, 1978). This occurs because uncharged, organic free bases are much more permeable than their protonated and charged

Received 24 August 1998

Revised 3 December 1998

Accepted 7 December 1998

Correspondence to: RJ Gillies counterparts and establish equal concentrations on both sides of the membrane (Gillies and Deamer, 1978). Since the ratio of charged-uncharged species increases with lower $\mathrm{pH}$, more total base is found in acidic compartments. This hypothesis is applicable to weakly basic chemotherapeutic drugs, such as doxorubicin $(\mathrm{pKa}=7.6)$, which is one of the most widely prescribed antineoplastic agents used in the treatment of breast cancer (Taylor et al, 1991).

\section{MATERIALS AND METHODS}

\section{Cell and tumour growth}

MCF-7 cells were obtained from W S Dalton (Arizona Cancer Center) and cultured in RPMI-1640/DMEM (Dulbecco's modified Eagle's medium) supplemented with $10 \%$ fetal bovine serum (FBS) (HyClone, Logan, UT, USA). For in vivo culturing, a suspension of $5 \times 10^{6} \mathrm{MCF}-7$ cells in $0.05 \mathrm{ml}$ of Hank's balanced salt solution were implanted in the mammary fat pads of 6- to 7 week-old female severe combined immunodeficient (SCID) mice. Since MCF-7 cells are oestrogen-dependent, 17 $\beta$-oestradiol pellets $(0.72 \mathrm{mg}, 60$-day release; Innovative Research of America, Sarasota, FL, USA) were subcutaneously implanted in the shoulder region of the mice by means of a 12-gauge trochar (Innovative Research) 2 days prior to tumour inoculation.

\section{Magnetic resonance spectroscopy}

Prior to MRS, mice were anaesthetized with a combination of ketamine $\left(72 \mathrm{mg} \mathrm{kg}^{-1}\right)$, xylazine $\left(6 \mathrm{mg} \mathrm{kg}^{-1}\right)$ and acepromazine (6 mg kg-1). A 3/4", 24-gauge catheter (Elf Sanofi Inc., Overland Park, KS, USA), connected to a $1.58 \mathrm{~mm}$ intradermal (i.d.)

*These authors contributed equally to this work. 
polyethylene tube (Becton Dickinson, Parsippany, NJ, USA), long enough to extend out of the magnet was inserted into the intraperitoneal (i.p.) cavity of the anaesthetized animal. The mouse was then immobilized on a home-built probe with a coil tunable to ${ }^{1} \mathrm{H}$ or ${ }^{31} \mathrm{P}$. After shimming the magnet, 3-APP $(0.15-0.3 \mathrm{ml}$, $128 \mathrm{mg} \mathrm{ml}^{-1}, \mathrm{pH}$ 7.4) was injected into the mouse via the i.p. catheter. Volume-selective ${ }^{31} \mathrm{P}$ spectra were acquired at $4.7 \mathrm{~T}$ on a Bruker Biospec with the ISIS sequence (Ordidge et al, 1988) with adiabatic slice-selective and excitation pulses repeated every $10-12 \mathrm{~s}$, using a gradient strength of $75 \mathrm{mT} \mathrm{m}^{-1}$. In all cases, a dwell time of $62.5 \mu$ s was employed, and 8192 data points were collected from 184 transients. Scout images of the tumour and surrounding abdominal tissue were obtained each time in order to guide the positioning of the voxel. The large spectral widths employed resulted in up to a $1 \mathrm{~mm}$ difference in the positioning of the voxel containing either $\alpha$-NTP or 3 -APP, and the voxel containing the central frequency. This chemical shift artefact was not corrected for, but voxel sizes and placement were chosen so as to minimize the contribution of signal arising from the mouse body wall, while covering as much of the tumour as possible.

\section{Measurement of intracellular $\mathrm{pH}$ in vitro}

Intracellular $\mathrm{pH}$ ( $\mathrm{pHi}$ ) was measured in vitro using the fluorescent dye, SNARF-1, as described previously (Martinez-Zaguilan et al, 1991). Briefly, MCF-7 cells were grown onto $9 \times 22 \mathrm{~mm}$ glass coverslips, washed three times with buffer A (1.3 mM calcium chloride, $1 \mathrm{~mm}$ magnesium sulphate, $5.4 \mathrm{~mm}$ potassium chloride, $0.44 \mathrm{mM} \mathrm{KH}_{2} \mathrm{PO}_{4}, 110 \mathrm{~mm}$ sodium chloride, $0.35 \mathrm{~mm} \mathrm{NaH} \mathrm{PO}_{4}$, $5 \mathrm{~mm}$ glucose, $2 \mathrm{~mm}$ glutamine and $5 \mathrm{~mm}$ HEPES, $5 \mathrm{~mm}$ MES, $10 \mathrm{~mm}$ sodium hydrogen carbonate $\left(\mathrm{NaHCO}_{3}\right)$ at a $\mathrm{pH}$ of 7.15 at $37^{\circ} \mathrm{C}$ and subsequently incubated for $30 \mathrm{~min}$ at $37^{\circ} \mathrm{C}$, in a $5 \%$ carbon dioxide atmosphere with $3 \mathrm{ml}$ of buffer A containing $10 \mu \mathrm{M}$ acetoxymethylester SNARF-1 (Molecular Probes, Eugene, OR, USA). This was followed by a second incubation in buffer A for $45 \mathrm{~min}$ to allow for complete hydrolysis of the dye. Coverslips were then placed in a holder/perfusion device and inserted into a fluorometer cuvette and fluorescence measurements were acquired at an excitation of $534 \mathrm{~nm}$ with the emission sequentially sampled at 584 and $644 \mathrm{~nm}$ in an SLM8000C (SLM, Urbana, IL, USA). The ratio (R) of fluorescence intensities of emissions at 584 and $644 \mathrm{~nm}$ was converted to $\mathrm{pHi}$ values using the equation: $\mathrm{pH}=7.38+\log _{10} 0.822+\log _{10}[(\mathrm{R}-0.458) /(1.928-\mathrm{R})]$. Data are presented as mean \pm s.e.m. of six independent measurements.

\section{Doxorubicin uptake and cytotoxicity in vitro}

MCF-7 cells were grown to confluence in 6-well plates at which time the cells were incubated in DMEM/F12 containing $20 \mathrm{mM}$ HEPES, $20 \mathrm{~mm}$ MES, $10 \% \mathrm{FBS}$ and $0.208 \mu \mathrm{Ci}$ per well of ${ }^{14} \mathrm{C}$ doxorubicin (Amersham) for $30 \mathrm{~min}$ at $37^{\circ} \mathrm{C}$ in a $5 \%$ carbon dioxide atmosphere at $\mathrm{pH}$ of 6.8 and 7.4 respectively. After the incubation, the plates were placed on ice and washed five times with ice-cold HBSS, followed by extraction with $1.0 \mathrm{ml}$ of $0.1 \mathrm{~N}$ $\mathrm{NaOH}$ for $1 \mathrm{~h}$ at $4^{\circ} \mathrm{C}$. Samples were divided into equal aliquots for determination of protein content using the Bradford assay (BioRad), and radioactivity using liquid scintillation counting.

Cytotoxicity was determined as previously described (Gillies et al, 1986). Briefly, cells were grown to log phase in 96-well plates and medium was exchanged for one at either $\mathrm{pH} 6.8$ or 7.4 containing the indicated concentrations of doxorubicin. Medium
Table 1 Theoretical and measured enhancement of doxorubicin uptake caused by increasing extracellular $\mathrm{pH}$ in vitro and in vivo

\begin{tabular}{lcc}
\hline In vitro & Low pHe & High pHe \\
\hline Extracellular $\mathrm{pH}$ & 6.8 & 7.4 \\
Intracellular $\mathrm{pH}(n)$ & $7.05 \pm 0.06(22)$ & $7.20 \pm 0.07(5)$ \\
Theoretical ratio & $0.69 \pm 0.07$ & $1.36 \pm 0.17$ \\
Theoretical enhancement & & $1.97 \pm 0.45$ \\
Doxorubicin uptake $(n)$ & $65.1 \pm 2.7(6)$ & $166.9 \pm 9.1(6)$ \\
Uptake enhancement & & $2.56 \pm 0.25$ \\
EC ${ }_{50}(\mu \mathrm{m})$ & 0.27 & 0.12 \\
Toxicity enhancement & & 2.25 \\
\hline In vivo & Control & $+\mathrm{NaHCO}_{3}$ \\
\hline pHe (800 mm ${ }^{3}$ tumour) & & $7.84 \pm 0.13$ \\
pHi $\left(800 \mathrm{~mm}^{3}\right.$ tumour) & $6.99 \pm 0.11$ & $7.39 \pm 0.13$ \\
pH gradient & $7.15 \pm 0.08$ & -0.45 \\
Theoretical ratio & +0.16 & 1.6 \\
Theoretical enhancement & 0.6 & 2.5 \\
\hline
\end{tabular}

Table 2 Tumour growth statistics

\begin{tabular}{llllc}
\hline & A & B & C & D \\
\hline No. mice & 17 & 13 & 12 & 13 \\
T-C $\left(\mathrm{d}\right.$ to $\left.300 \mathrm{~mm}^{3}\right)$ & - & - & 15 & 22 \\
T/C $(\%)$ & - & - & 0.47 & 0.29 \\
Growth rate $\left(\mathrm{d}^{-1}\right)$ & 0.206 & 0.215 & 0.156 & 0.092 \\
Standard deviation & 0.094 & 0.060 & 0.067 & 0.047 \\
$P$ vs group A & - & 0.732 & 0.031 & 0.002 \\
$P$ vs group B & 0.732 & - & 0.027 & 0.001 \\
$P$ vs group C & 0.031 & 0.027 & - & 0.008 \\
$P$ vs group D & 0.002 & 0.001 & 0.008 & - \\
& & & & \\
\hline
\end{tabular}

$\mathrm{pH}$ was buffered using non-volatile buffers (10 mM MES, $20 \mathrm{~mm}$ HEPES and $10 \mathrm{mM}$ TRICINE) in combination with bicarbonate concentrations that were adjusted to be in equilibrium with $5 \%$ ambient carbon dioxide. Twenty-four hours later, regular growth medium was replaced and cells allowed to grow a subsequent $72 \mathrm{~h}$, after which time they were fixed and stained with crystal violet for determination of cell number.

\section{Calculation of theoretical drug distributions}

Cytoplasmic-extracellular drug ratios were calculated at the steady-state using methods described previously (Roos, 1978; Raghunand et al, 1999b). Briefly, the ratio of protonated (charged) to deprotonated (uncharged) doxorubicin were calculated from the Henderson-Hasselbach equation in both intracellular and extracellular compartments using the $\mathrm{pHi}$ and $\mathrm{pHe}$, respectively, and a drug $\mathrm{pKa}$ of 7.6. The concentration of the uncharged specie was set to 1.0 on both sides of the membrane and the total concentration was calculated as the sum of (charged + uncharged). Data were expressed as a ratio of intra- to extracellular concentrations. Enhancement was calculated as the ratio at high $\mathrm{pH}$, relative to that at low $\mathrm{pH}$.

\section{Tumour growth statistics}

Tumour size was measured with calipers and calculated as: $\left[(\text { width })^{2} \times\right.$ length $] / 2$. Data were linearized by converting volumes (in $\mathrm{mm}^{3}$ ) to their cube roots. The least squares regression line of 

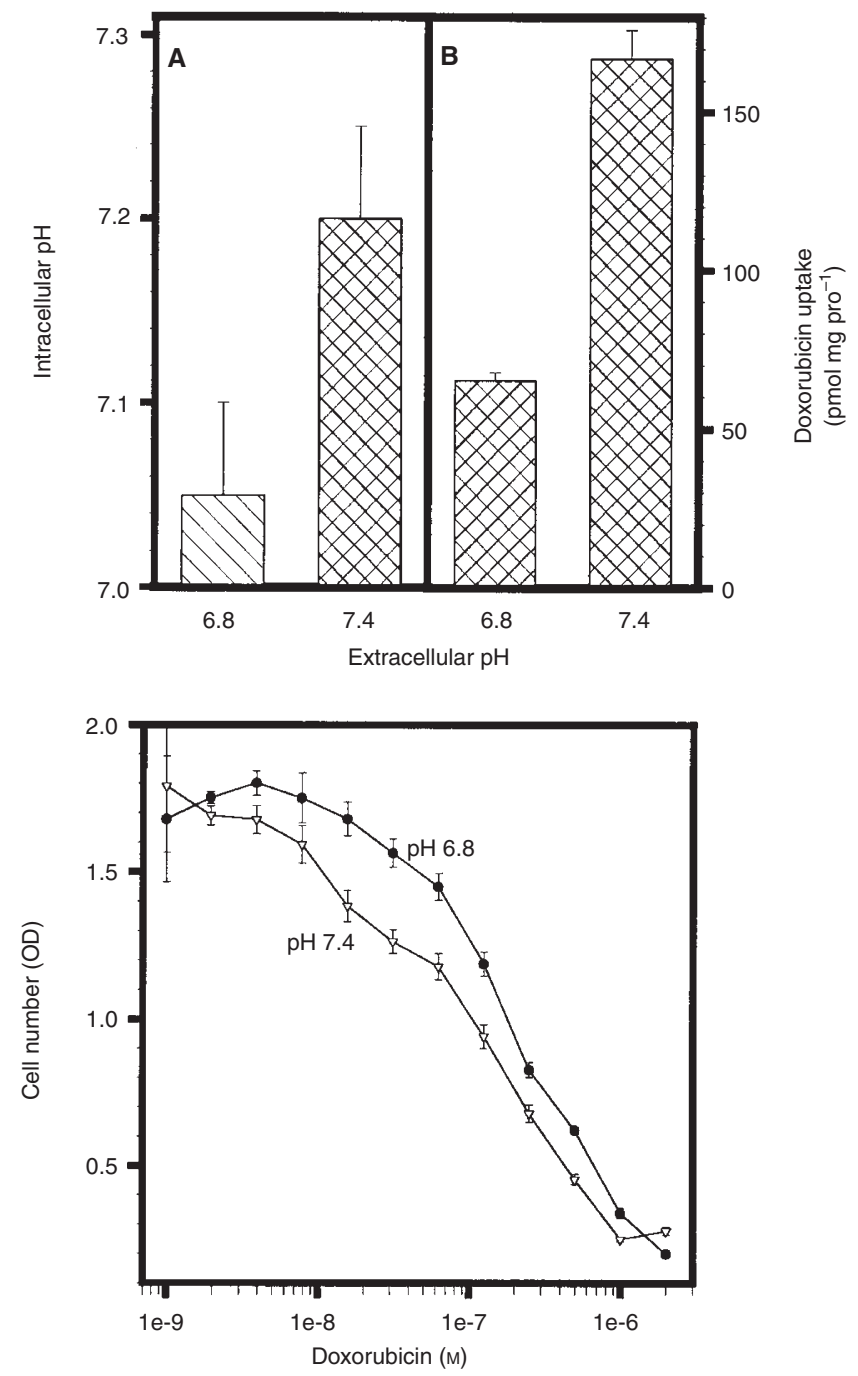

Figure 1 In vitro effects of $\mathrm{pH}$ on doxorubicin chemotherapy. (A) Intracellular pH of MCF-7 cells in-vitro. MCF-7 cells grown on coverslips were loaded with SNARF-1 fluorescent dye and the intracellular $\mathrm{pH}$ was measured as described in Methods. (B) Doxorubicin levels in MCF-7 cells as a function of pHe. MCF-7 cells were incubated with ${ }^{14} \mathrm{C}$ doxorubicin for $30 \mathrm{~min}$ at medium $\mathrm{pH}$ of 6.8 or 7.4 , after which time cells were washed and radioactivity determined (see Methods). Data are expressed as pmol mg protein $^{-1}$. (C) Cytotoxicity of doxorubicin as a function of pHe. MCF-7 cells were grown in 96-well plates to sub-confluence, at which time they were treated with doxorubicin at the indicated concentrations. Ninety-six hours later, media were removed and remaining cell numbers were determined by crystal violet staining, as described previously (Gillies et al, 1986). Data are shown for MCF-7 cells at pH 6.8 and 7.4. OD, optical density

the cube root volumes from day 9 onward were fit for each mouse with non-zero tumour growth. Three-way analyses of variance were used to examine the effect of different experiments, injection (doxorubicin vs saline), and water (plain vs bicarbonate). The differences between experiments were not significant $(P=0.767)$ and, hence, data from all experiments were pooled for further twoway analyses of variance. Tumour cell $\log$ kill was calculated from the equation $\left[(\mathrm{T}-\mathrm{C}) / 3.32 \mathrm{~T}_{\mathrm{d}}\right]$, where $\mathrm{T}$ and $\mathrm{C}$ are the number of days it took for treated and control groups, respectively, to reach $1000 \mathrm{~mm}^{3} . \mathrm{T}_{\mathrm{d}}$ is the exponential doubling time of the treated group (Corbett et al, 1982). T/C was calculated as the ratio of the median volumes of treated and control groups on day 20 . T/C $<44 \%$ is considered significant by the Division of Cancer Treatment (National Cancer Institute), while a T/C value of $<10 \%(\mathrm{DN}-2$ level activity) is considered highly significant.

\section{RESULTS}

\section{$\mathrm{pH}$ affects doxorubicin distribution and cytotoxicity in vitro}

Solid tumours generally exhibit pHe values of 6.7-6.8 (WikeHooley et al, 1984; Griffiths, 1991; Gillies et al, 1994; McCoy et al, 1995; Raghunand et al, 1999a). In vitro, MCF-7 cells at medium $\mathrm{pH}$ values of 6.8 and 7.4 have $\mathrm{pHi}$ values of $7.05 \pm 0.06$ and $7.20 \pm 0.07$, respectively, as measured with fluorescence (Figure 1A). Thus, according to theory (see Methods), the $\mathrm{pH}$ gradient of +0.25 at $\mathrm{pHe}=6.8$ will yield an intracellular-extracellular doxorubicin ratio of $0.69 \pm 0.07$, whereas at $\mathrm{pHe}=7.4$, the ratio will be $1.36 \pm 0.17$ (Table 1). Hence, there will be a $1.97 \pm 0.45$-fold enhancement of the steady-state intracellular drug concentration as $\mathrm{pHe}$ is raised from 6.8 to 7.4. The empirical steady-state levels of ${ }^{14} \mathrm{C}$ doxorubicin in $\mathrm{MCF}-7$ cells at medium $\mathrm{pH}$ values of 6.8 and 7.4 show a $2.56 \pm 0.25$-fold enhancement (Figure 1B), which is in good agreement with theory.

The difference in intracellular levels of doxorubicin between low and high pHe values should give rise to differences in cytotoxicity. Figure $1 \mathrm{C}$ shows that, following a 24-h treatment, the $\mathrm{EC}_{50}$ of doxorubicin in MCF-7 cells is $0.27 \mu \mathrm{m}$ at $\mathrm{pH} 6.8$, compared to $0.12 \mu \mathrm{m}$ at $\mathrm{pH}$ 7.4. One-hour treatments with doxorubicin yielded essentially the same results, although both $\mathrm{EC}_{50}$ values were higher. Similar data for doxorubicin have been reported elsewhere (Tannock and Rotin, 1989; Simon and Schindler, 1994). In the current study, the 2.25-fold enhancement of doxorubicin cytotoxicity at $\mathrm{pH} 7.4$ compared to $\mathrm{pH} 6.8$ is similar in magnitude to both theory and the empirical doxorubicin distribution ratio (cf. Table 1). Thus, the enhanced cytotoxicity at high pHe is likely due to increased partitioning of doxorubicin into cells.

\section{Bicarbonate raises $\mathrm{pHe}$ in vivo}

While there is substantial evidence that doxorubicin is more effective at higher $\mathrm{pHe}$, this phenomenon is of limited utility unless the $\mathrm{pHe}$ of tumours can be manipulated in vivo. For the current experiments, $\mathrm{pHe}$ was raised via sodium bicarbonate-induced metabolic alkalosis. However, the addition of bicarbonate could have important negative sequelae, since it is osmotically active and could cause hypernatraemia. To determine the maximum tolerated doses of bicarbonate, eight different cohorts of SCID mice were given $\mathrm{NaHCO}_{3}$-supplemented water to drink ad libitum. Bicarbonate concentrations ranged from 0 to $250 \mathrm{mM}$. Animal weights and water consumption were monitored daily. For bicarbonate concentrations up to $225 \mathrm{~mm}$, weight gain and subjective parameters (e.g. grooming behaviour and activity) were unaffected compared to water controls for up to 9 weeks (data not shown). In a separate experiment, animals were given $200 \mathrm{~mm} \mathrm{NaHCO}_{3}$ ad lib. Four to 10 days following commencement of bicarbonate therapy, $\mathrm{pHi}$ and $\mathrm{pHe}$ were measured using ${ }^{31} \mathrm{P}$ MRS. A representative ${ }^{31} \mathrm{P}$ spectrum from an untreated MCF-7 cell tumour is shown in Figure 2A. In this Figure, the pHe measured by 3-APP is 6.57 and the pHi measured by $\mathrm{Pi}$ is 6.95 . Figure $2 \mathrm{~B}$ shows the region containing the 3-APP resonance from a representative ${ }^{31} \mathrm{P}$ MRS spectrum of 


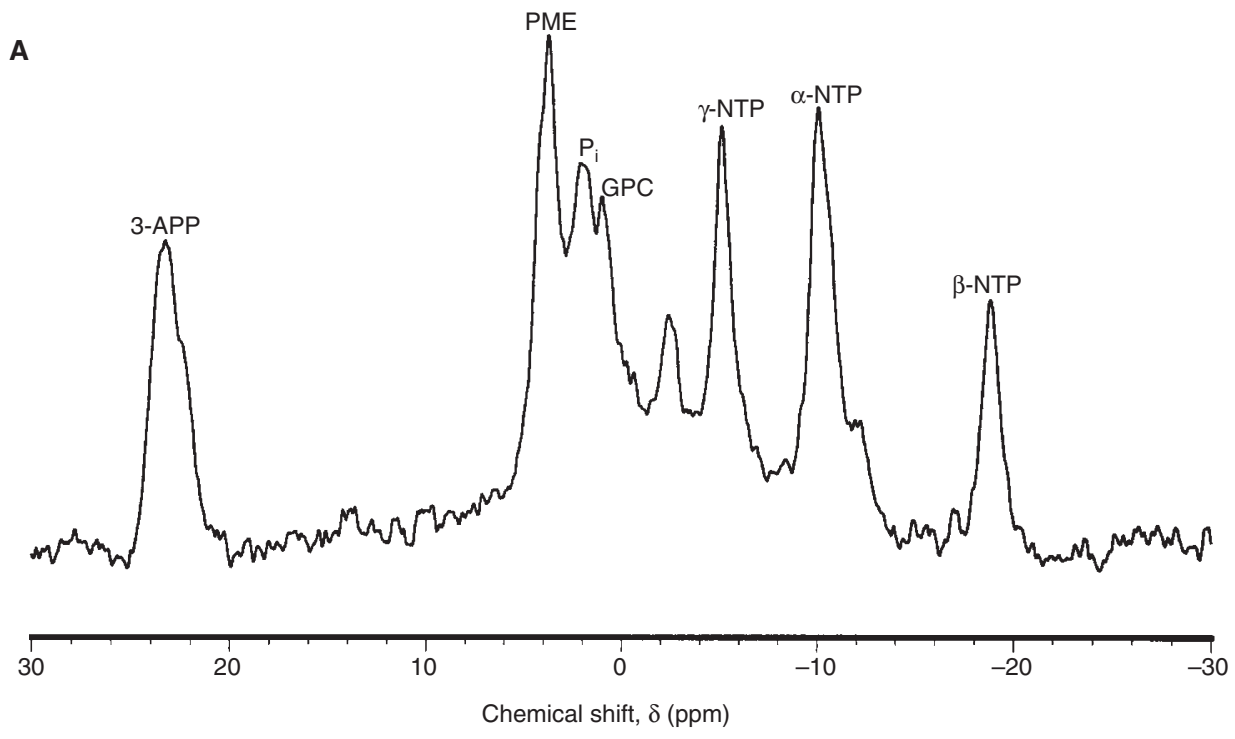

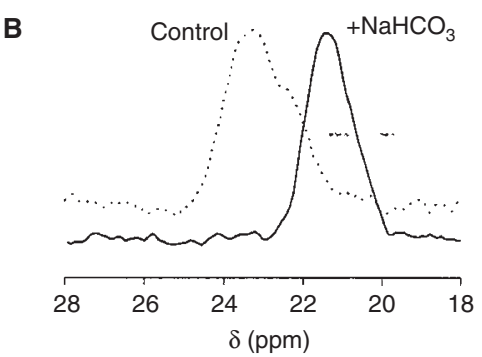

MCF-7 tumours obtained 4 days following substitution of drinking water with $200 \mathrm{~mm} \mathrm{NaHCO}$. Note the shift in the position of the 3-APP resonance relative to controls indicating a significant alkalinization of pHe. All other resonances ( $\mathrm{Pi}, \mathrm{PME}, \mathrm{ATP})$ were statistically indistinguishable from the controls. In this example, the measured $\mathrm{pHe}$ was 7.72 . Thus, $\mathrm{NaHCO}_{3}$ can significantly increase $\mathrm{pHe}$.

Results from all of the MRS-based $\mathrm{pH}$ measurements are shown in Figure 3A, expressed as a function of tumour size. Note that in untreated MCF-7 tumours (triangles), the pHe (open) and pHi (closed) decrease with approximately the same slope with increasing tumour size. Thus, the $\mathrm{pH}$ gradient is relatively constant at $0.22 \pm 0.09 \mathrm{pH}$ unit, consistent with previous reports (Raghunand et al, 1999a). In animals supplemented with $\mathrm{HCO}_{3}^{-}$, the measured $\mathrm{pHe}$ values (open circles) are significantly higher than the measured pHi values (closed circles). This is significantly different than the $\mathrm{pHe}$ and $\mathrm{pHi}$ of control animals. The direction of the plasmalemmal (in-out) $\mathrm{pH}$ gradient is shown by arrows in the Figure and it is clear that the direction is reversed in all $\mathrm{NaHCO}_{3}$ treated animals, compared to controls. The pHe reported by the 3 APP resonance in $\mathrm{NaHCO}_{3}$-treated tumours is high, $7.84 \pm 0.13$ $\mathrm{pH}$ unit. The $\mathrm{pHi}$ values in the same animals were $7.39 \pm 0.12$. The imprecision in both the $\mathrm{pHe}$ and $\mathrm{pHi}$ measurements are likely higher due to these values being near the alkaline extreme of the titration curves for both 3-APP and Pi respectively. Nevertheless, the data clearly show that chronic bicarbonate therapy effectively
Figure 2 Measurement of tumour pH with ${ }^{31} \mathrm{P}$ MRS. (A) In vivo ${ }^{31} \mathrm{P}$ NMR spectrum of MCF-7 tumour grown in mammary fat pad of severe combined immunodeficient (SCID) mouse. The in vivo ${ }^{31} \mathrm{P}$ spectra were processed by Fourier transformation with $15 \mathrm{~Hz}$ exponential line broadening and referenced to the $\alpha$-resonance of NTP at $-10.05 \mathrm{ppm}$. The intra and extracellular $\mathrm{pH}$ values were obtained from the chemical shifts of $\mathrm{Pi}$ and 3 APP respectively (McCoy et al, 1995; Raghunand et al, 1999a). Pi, inorganic phosphate; NTP, nucleoside triphosphates; PME, phosphomonoesters. (B) Downfield region of spectra from control and $\mathrm{NaHCO}_{3}$-treated mice. Downfield region of ${ }^{31} \mathrm{P}$ MR spectra showing 3-APP resonance from MCF-7 tumours in SCID mice. The dotted line (downfield resonance) is from control mouse at pHe 6.57 and the solid line (upfield resonance) is from mouse given $200 \mathrm{~mm} \mathrm{NaHCO}_{3}$ ad libitum for 4 days, showing a pHe of 7.72 . Acquisition conditions were identical to those in Figure $1 \mathrm{~A}$

alkalinizes the $\mathrm{pHe}$ in vivo, generating an (in-out) $\Delta \mathrm{pH}$ of approximately $-0.45 \mathrm{pH}$ unit, compared to a $\Delta \mathrm{pH}$ of +0.20 in untreated animals.

\section{Bicarbonate chemosensitizes tumour xenografts in vivo}

According to the measured $\mathrm{pH}$ gradients, the theoretical cytosolic-extracellular drug ratio should be 1.6 in bicarbonatetreated animals, and 0.6 in controls, leading to a 2.5 -fold increase in doxorubicin sensitivity (Table 1). To test this, MCF-7 tumours were grown in the mammary fat pads of 6-week-old female SCID mice, to sizes of $50-200 \mathrm{~mm}^{3}$, and were forcibly randomized according to tumour size into four groups.

Groups A and C were given normal water ad libitum, while the water given to groups B and D was supplemented with $200 \mathrm{~mm}$ $\mathrm{NaHCO}_{3}$. The start of the bicarbonate treatment was designated as day 1 . On days 3, 7 and 11, animals in groups $\mathrm{C}$ and $\mathrm{D}$ were injected i.p. with doxorubicin while animals in groups $\mathrm{A}$ and $\mathrm{B}$ ( $n=10$ each) received saline injections of the same volume, as per established protocols (Paine-Murrieta et al, 1997). The maximum tolerated dose (MTD) of SCID mice for doxorubicin under this regimen is a cumulative $6.7 \mathrm{mg} \mathrm{kg}^{-1}$ (Paine-Murrieta et al, 1997). Initial experiments used three doses of doxorubicin at $2.0 \mathrm{mg} \mathrm{kg}^{-1}$ (cohorts $\mathrm{C}_{1} / \mathrm{D}_{1}$ ) and this was reduced in later experiments to three doses at $1.6 \mathrm{mg} \mathrm{kg}^{-1}$ (cohorts $\mathrm{C}_{2} / \mathrm{D}_{2}$ ) to reduce morbidity. In all 

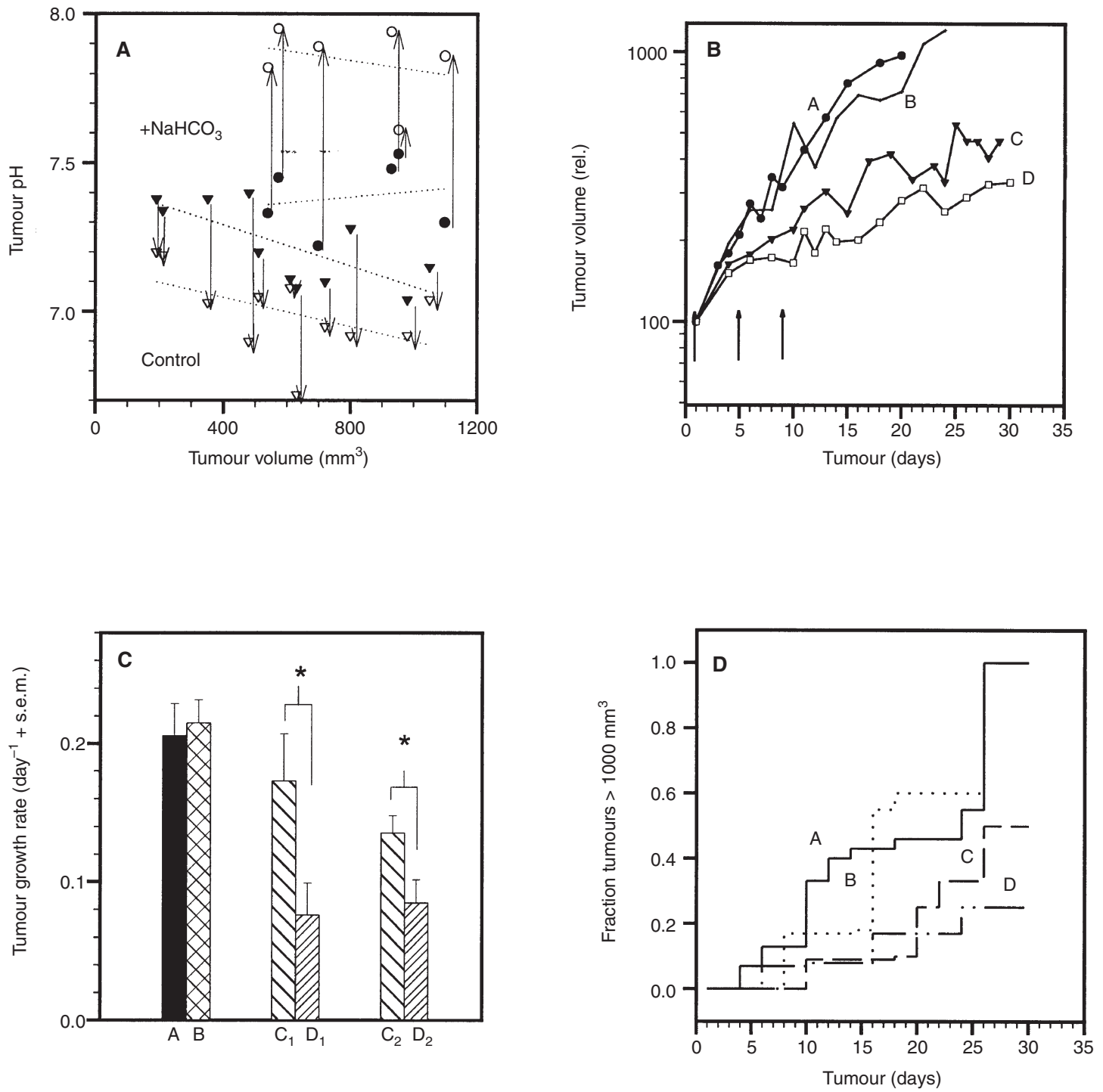

Figure 3 In vivo effects of combination chemotherapy. (A) pHi and pHe of MCF-7 tumours in control (triangles) and bicarbonate-treated (circles) mice. pHi and $\mathrm{pHe}$ values were measured using ${ }^{31} \mathrm{P}$ MRS, as described for Figure 1. These pHe data were collected 4-10 days following commencement of bicarbonate therapy. Closed symbols represent the intracellular $\mathrm{pH}$ values, measured with $\mathrm{Pi}$, and the open symbols represent extracellular pH values, measured with 3APP. Arrows indicate the direction of the intra- to extracellular gradient, and dotted lines represent independent linear regressions of the four data sets. (B) Tumour volumes as a function of time. MCF-7 tumours were grown in SCID mice to volumes of 50-200 $\mathrm{mm}^{3}$, after which time they were randomized into four cohorts (A-D). Beginning on day 1, cohorts A and C received drinking water, whereas cohorts B and D received 200 mM NaHCO. On days 1,5 and 9 , animals of cohorts $C$ and $D$ were injected i.p. with doxorubicin (arrows), whereas animals from cohorts $A$ and B were injected with saline. On day 15, animals of cohorts $B$ and $D$ were placed back on drinking water. For the purpose of display, tumour volumes were normalized to $100 \%$ on day 4 . Data points represent the pooled means of 3-16 measurements at each time point from all experiments. (C) Specific growth rates. The rate of increase in tumour size was determined between days 9 and 15 for each animal by linear regression and expressed as fraction of surviving mice per day individual tumour. The illustrated data represent the mean \pm standard deviation of these data sets binned by cohort. The cohorts were as described in Figure $3 B$, except that cohorts $C_{1} / D_{1}$ and $C_{2} / D_{2}$ were separated since they received doxorubicin at 2.0 and $1.6 \mathrm{mg} \mathrm{kg}^{-1}$ respectively. ${ }^{*}$ Indicates values were significantly different $(P<0.03)$ by Student's $t$-test. (D) Individual tumour growth curves. Percent of total tumours in a cohort greater than $1000 \mathrm{~mm}^{3}$ are plotted as a function of time since beginning of experiments. Cohorts are as described for Figure 3B

experiments, the incidence of morbidity following drug treatment was identical between groups $\mathrm{C}$ and $\mathrm{D}$. In parallel experiments, three doses of i.p. doxorubicin at $2.0 \mathrm{mg} \mathrm{kg}^{-1}$ on days 1,5 and 9 gave no evidence of vesication upon day 10 necropsy. Tumour volumes and animal weights were monitored every 2-3 days. For graphing purposes, tumour volumes for each mouse were normalized to day 1. As shown in Figure 3B, doxorubicin has a significant effect on the tumour growth rate, and this effect is greater in animals co-treated with bicarbonate. For statistical analyses, normalized data were used to calculate specific tumour growth rates for each tumour, from day 9 onward. The means of these normalized tumour growth rates are shown in Figure 3C. Regardless of dose, the tumour growth rate was significantly $(P \leq$ $0.03)$ slower in the bicarbonate plus doxorubicin groups $\left(\mathrm{D}_{1}, \mathrm{D}_{2}\right)$, compared to the doxorubicin alone groups $\left(\mathrm{C}_{1}, \mathrm{C}_{2}\right)$ (Table 2 ). Furthermore, bicarbonate alone had no significant effect on the growth rate $(P>0.4, \mathrm{~A}$ vs $\mathrm{B})$. Table 2 also shows that the tumour growth delays $(\mathrm{T}-\mathrm{C})$ were 15 and 22 days at $300 \mathrm{~mm}^{3}$ for groups $\mathrm{C}$ and $\mathrm{D}$ respectively. However, since the slopes of the growth curves diverge, the $\mathrm{T}-\mathrm{C}$ increased with time, and thus cannot be used to 
reliably calculate log cell kill. The $\mathrm{T} / \mathrm{C}$ values calculated at day 20 were $0.47(751 / 1571)$ and $0.29(458 / 1571)$ for groups $C$ and D respectively. A modified (Kaplan-Meier type) survival plot showing the fraction of remaining animals with small $\left(<1000 \mathrm{~mm}^{3}\right)$ tumours is shown in Figure 3D. These data show that animals receiving doxorubicin plus bicarbonate take significantly longer to develop larger tumours compared to the other groups.

\section{DISCUSSION}

These data indicate that raising pHe increased the cytotoxic effectiveness of doxorubicin both in vitro and in vivo. In-vitro, raising the pHe from 6.8 to 7.4 resulted in a 2.25 -fold enhancement of cytotoxicity and a 2.56-fold increase in intracellular doxorubicin concentrations. These data compared well to the 1.97-fold enhancement predicted from the calculated difference in doxorubicin distributions (Table 1). It is possible that the larger enhancement observed empirically, relative to theory, is due to sequestration of doxorubicin in acidic vesicles, which was not accounted for in the theoretical calculations (Altan et al, 1998; Raghunand et al, 1999b).

In vivo, in the SCID mouse-MCF7 tumour model system used here, the data clearly show that $\mathrm{NaHCO}_{3}$ effectively raises the $\mathrm{pHe}$ for extended periods of time. Prolonged treatments with bicarbonate in drinking water did not have significant effects on animal well-being.

Nevertheless, for bicarbonate therapy to have clinical utility, the minimum required treatment time needs to be determined. Previous protocols involving bicarbonate treatment of humans have primarily been developed to counteract acidosis caused by exercise or renal failure (Passfall et al, 1997; Verbitsky et al, 1997). A problem in these protocols is poor patient compliance, with chronic bicarbonate ingestion. Clinical metabolic alkalinization with bicarbonate will be most successful if it is administered acutely, viz. shortly prior to the time of chemotherapy. Hence, experiments are underway to determine the time course of alkalinization and whether acute, intermittent alkalinization is as effective, compared to chronic bicarbonate administration.

Combination therapy with bicarbonate and doxorubicin resulted in a significantly lowered tumour growth rate compared to treatment with doxorubicin alone. In SCID mice, as in human patients, doxorubicin alone is not a highly effective cytotoxic agent with much heterogeneity of response. Many workers have used SCID mice to test doxorubicin chemotherapy (Liu et al, 1996; Cesano et al, 1997; Durand and LePard, 1997). However, a recent report indicates that doxorubicin is not effective to treat tumours in SCID mice, due to granulocyte toxicity to the host (Polin et al, 1997). This may be observed in our studies, since the tumour outgrowth did not occur with the same doubling time as the untreated tumours. Consequently, log kill values could not be reliably calculated. However, the T/C value measured at day 20 was 0.29 for group D, compared to 0.47 for group $\mathrm{C}$. $\mathrm{T} / \mathrm{C}<44 \%$ is considered significant by the Division of Cancer Treatment (NCI). Thus, this measure indicates a meaningful boost in therapeutic effectiveness caused by raising the $\mathrm{pH}$ with bicarbonate. In other systems, such as sygeneic mice (16/C mammary adenocarcinoma in $\mathrm{C} 3 \mathrm{H}$ mice), log kill from doxorubicin can be 2.5-3.0 ( $\mathrm{T}$ Corbett, personal communication). Hence, experiments are planned using this more responsive mouse system, even though it is not a high connectivity model for human clinical response.
The growth rates resulting from the two different doxorubicin doses were not significantly different (i.e. $C_{1}$ vs $C_{2}, D_{1}$ vs $D_{2}$ ). However, the lower doxorubicin dose did reduce the mortality of the treated animals during the course of the experiments. At $2 \mathrm{mg} \mathrm{kg}^{-1}$, half of the animals survived in both groups to day 30 . At $1.6 \mathrm{mg} \mathrm{kg}^{-1}$, only one animal from each group (out of eight) did not survive until day 30. It must be noted that doxorubicin-treated animals appeared more distressed than controls. By day 15, animals in groups $\mathrm{C}$ and $\mathrm{D}$ displayed poor grooming and urinary discharges. By day 30, all animals in group D were experiencing anal discharge as well. This suggests that, in addition to the tumours, bicarbonate also sensitized the intestinal epithelium to doxorubicin cytotoxicity. Since bicarbonate should only have this effect on tissues with low initial $\mathrm{pHe}$, this might indicate that intestinal epithelial cells or colonic crypts have a naturally low pHe. This side-effect of combination bicarbonate-doxorubicin chemotherapy might be avoided with shorter bicarbonate treatment times, more selective alkalinizing agents, or protection of the intestinal epithelium with acid buffers or V-ATPase inhibitors. Despite the qualitatively poorer condition of group D animals, there was no significant difference in their body weights, compared to animals of group C.

Our calculations predict that alkalinization should provide a two- to threefold increase in the therapeutic efficacy of doxorubicin. This is significant, because many current clinical studies indicate that threefold accelerated dosing of doxorubicin is more effective in treating node-positive breast cancers (Bearman et al, 1997). However, doxorubicin has associated cardiotoxicity and there is a significant morbidity associated with accelerated dosing regimens. Thus, if two- to threefold increases in cytosolic doxorubicin could be achieved through manipulation of $\mathrm{pH}$, it is likely that the therapeutic benefits could be realized without the increased morbidity associated with accelerated dosing.

In summary, the current data indicate that the pHe of human breast tumour xenografts is acidic and that this reduces the cytotoxicity of doxorubicin in vitro and in vivo. Tumour pHe can be chronically raised by treatment with sodium bicarbonate in the drinking water of tumour-bearing SCID mice, and this significantly increases the chemotherapeutic efficacy of doxorubicin chemotherapy in vivo.

\section{ACKNOWLEDGEMENTS}

The authors would like to thank J-P Galons for helpful discussions, L Celaya for secretarial assistance, and TH Corbett, J Evelhoch and S Salmon for thoughtful evaluations of the manuscript. Support from the US Army Breast Cancer Initiative grants DAMD-17-94-J-4368 (RJG) and DAMD-17-96-1-6131 (ZMB) and NIH RO1-CA23074 is also gratefully acknowledged.

\section{REFERENCES}

Altan N, Chen Y, Schindler M and Simon SM (1998) Defective acidification in human breast tumor cells and implications for chemotherapy. J Exp Med 187: 1583-1598

Bearman SI, Overmoyer BA, Bolwell BJ, Taylor CW, Shpall EJ, Cagnoni PJ, Mechling BE, Ronk B, Baron AE, Purdy MH, Ross M and Jones RB (1997) High-dose chemotherapy with autologous peripheral blood progenitor cell support for primary breast cancer in patients with 4-9 involved axillary lymph nodes. Bone Marrow Transplant 20: 931-937

Cesano A, Visonneau S, Rovera G and Santoli (1997) Synergistic effects of adriamycin and TALL-104 cell therapy against a human gastric carcinoma in vivo. Anticancer Res 17: 1887-1892 
Corbett TH, Roberts BJ, Trader MW, Laster WR Jr, Griswold DPJ and Schabel FM Jr. (1982) Response of transplantable tumors of mice to anthracenedione derivatives alone and in combination with clinically useful agents. Cancer Treat Rep 66: 1187-1200

Durand RE and LePard NE (1997) Tumour blood flow influences combined radiation and doxorubicin treatments. Radiother Oncol 42: 171-179

Gillies RJ and Deamer DW (1978) Intracellular pH: methods and applications. Curr Top Bioenergetics 9: 63-87

Gillies RJ, Didier N and Denton M (1986) Determination of cell number in monolayer cultures. Analyt Biochem 159: 109-113

Gillies RJ, Liu Z and Bhujwalla Z (1994) ${ }^{31} \mathrm{P}-\mathrm{MRS}$ measurements of extracellular $\mathrm{pH}$ of tumors using 3-aminopropylphosphonate. Am J Physiol 195-203

Griffiths JR (1991) Are cancer cells acidic? Br J Cancer 64: 425-427

Liu C, Lambert JM, Teicher BA, Blattler WA and O'Connor R (1996) Cure of multidrug-resistant human B-cell lymphoma xenografts by combinations of anti-B4-blocked ricin and chemotherapeutic drugs. Blood 87: 3892-3898

Martinez-Zaguilan R, Martinez GM, Lattanzio F and Gillies RJ (1991) Simultaneous measurement of intracellular $\mathrm{pH}$ and $\mathrm{Ca}^{2+}$ using the fluorescence of SNARF-1 and Fura-2. Am J Physiol 260: C297-C307

McCoy CL, Parkins CS, Chaplin DJ, Griffiths JR, Rodrigues LM and Stubbs M (1995) The effect of blood flow modification on ontra- and extracellular $\mathrm{pH}$ measured by $31 \mathrm{P}$ magnetic resonance spectroscopy in murine tumours. $\mathrm{Br} \mathrm{J}$ Cancer 72:

Negendank W (1992) Studies of human tumors by MRS: a review. NMR Biomed 5: 303-324

Ordidge RJ, Bowley RM and McHale G (1988) A general approach to selection of multiple cubic volume elements using the ISIS technique. Magn Reson Med $\mathbf{8}$ 323-331

Paine-Murrieta GD, Taylor CW, Curtis RA, Lopez MHA, Dorr RT, Johnson CS, Funk CY, Thompson F and Hersh EM (1997) Human tumor models in the severe combined immune deficient (Scid) mouse. Cancer Chemother Pharmacol $\mathbf{x x}$ : $1-6$

Passfall J, Pai J, Spies KP, Haller H and Luft FC (1997) Effect of water and bicarbonate loading in patients with chronic renal failure. Clin Nephrol 47: 92-98

Polin L, Valeriote F, White K, Panchapor C, Pugh S, Knight J, LoRusso, Hussain M, Liversidge E, Peltier N, Golakoti T, Patterson G, Moore and Corbett TH (1997) Treatment of human prostate tumors PC-3 and TSU-PR1 with standard and investigational agents in SCID mice. Invest New Drugs 15: 99-108

Raghunand N, Altbach MI, Van Sluis R, Baggett B, Taylor CW, Bhujwalla ZM and Gillies RJ (1999a) Plasmalemmal pH-gradients in drug-sensitive and drugresistant MCF-7 human breast carcinoma xenografts measured by 31P MR spectroscopy. Biopharm Pharmacol 57: 309-312

Raghunand N, Martinez-Zaguilan R, Wright SH and Gillies RJ (1999b) pH and drug resistance II: turnover of acidic vesicles and resistance to weakly basic chemotherapeutic drugs. Biochem Pharmacol 57: 1047-1058

Roos A (1978) Weak acids, weak bases, and intracellular pH. Respir Physiol 33: $27-30$

Simon SM and Schindler M (1994) Cell biological mechanisms of multidrug resistance in tumors. Proc Natl Acad Sci USA 91: 3497-3504

Tannock IF and Rotin D (1989) Acid pH in tumors and its potential for therapeutic exploitation. Cancer Res 49: 4373-4384

Taylor CW, Dalton WS, Parrish PR, Gleason MC, Bellamy WT, Thompson FH, Roe DJ and Trent JM (1991) Different mechanisms of decreased drug accumulation in doxorubicin and mitoxantrone resistant variants of the MCF7 human breast cancer cell line. Br J Cancer 63: 923-929

Verbitsky O, Mizrahi J, Levin M and Isakov E (1997) Effect of ingested sodium bicarbonate on muscle force, fatigue, and recovery. J Appl Physiol 83: 333-337 Warburg O (1956) On the origin of cancer cells. Science 123: 309-314

Wike-Hooley JL, Haveman J and Reinhold HS (1984) The relevance of tumour pH to the treatment of malignant disease. Radiother Oncol 2: 343-366 